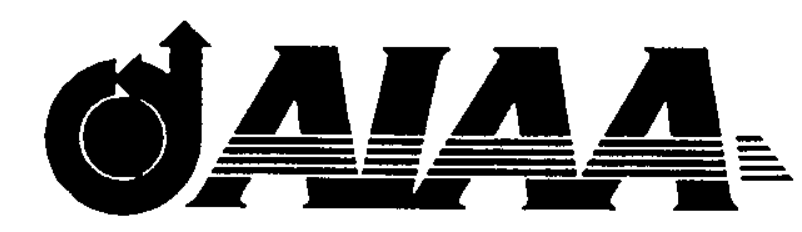

\title{
AIAA 95-0049
}

A New Implicit Algorithm with Multigrid for Unsteady Incompressible Flow Calculations

A. Belov, L. Martinelli, and A. Jameson

Princeton University

Princeton, NJ

\section{3rd Aerospace Sciences Meeting and Exhibit January 9-12, 1995 / Reno, NV}

For permission to copy or republish, contact the American Institute of Aeronautics and Astronautics 370 L'Enfant Promenade, S.W., Washington, D.C. 20024 


\title{
A New Implicit Algorithm with Multigrid for Unsteady Incompressible Flow Calculations
}

\author{
Andrey Belov ${ }^{*}$ Luigi Martinelli, ${ }^{\dagger}$ and Antony Jameson ${ }^{\ddagger}$ \\ Department of Mechanical and Aerospace Engineering \\ Princeton University \\ Princeton, New Jersey 08544 U.S.A.
}

\section{Abstract}

We present a new fully-implicit algorithm for unsteady incompressible flow calculations for both the Euler and Navier-Stokes equations. The new method couples the artificial compressibility approach with an implicit A-stable discretization of the unsteady terms in order to advance the solution in a time-accurate manner with no stability limitations on the time step. A pseudotransient steady state problem is solved at each time step to provide a direct coupling between the velocity and pressure fields, and to satisfy the divergence-free constraint. The present algorithm solves the pseudotransient problem by using the highly efficient multigrid time stepping technique, originally developed by Jameson [1] for compressible flow calculations. Both viscous and inviscid test problems are presented. An inviscid two-dimensional flow over an oscillating cylinder is used to validate the method by comparison with analytic results. The mean quantities of the unsteady viscous flow over a circular cylinder for $R e \leq 200$ are computed and found to be in good agreement with the computational and experimental data obtained by other authors. Results for the unsteady viscous flow over a NACA0012 airfoil at $20^{\circ}$ angle of attack are also presented, and domain truncation and time resolution effects are discussed.

\section{Nomenclature}

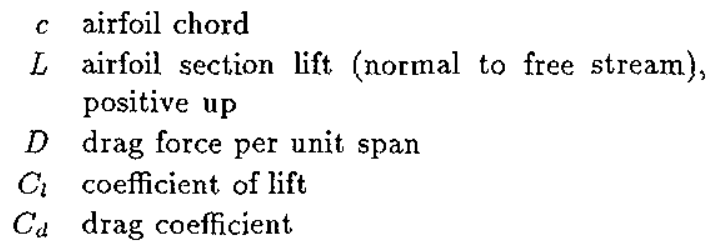

Copyright @1995 by the American Institute of Aeronautics and Astronautics, Inc. All rights reserved

* Ph.D. Student

†Professor of Aerospace Engineering, Member of AIAA

$\ddagger$ James S. McDonnell Distinguished University Professor of Aerospace Engineering, AIAA Fellow

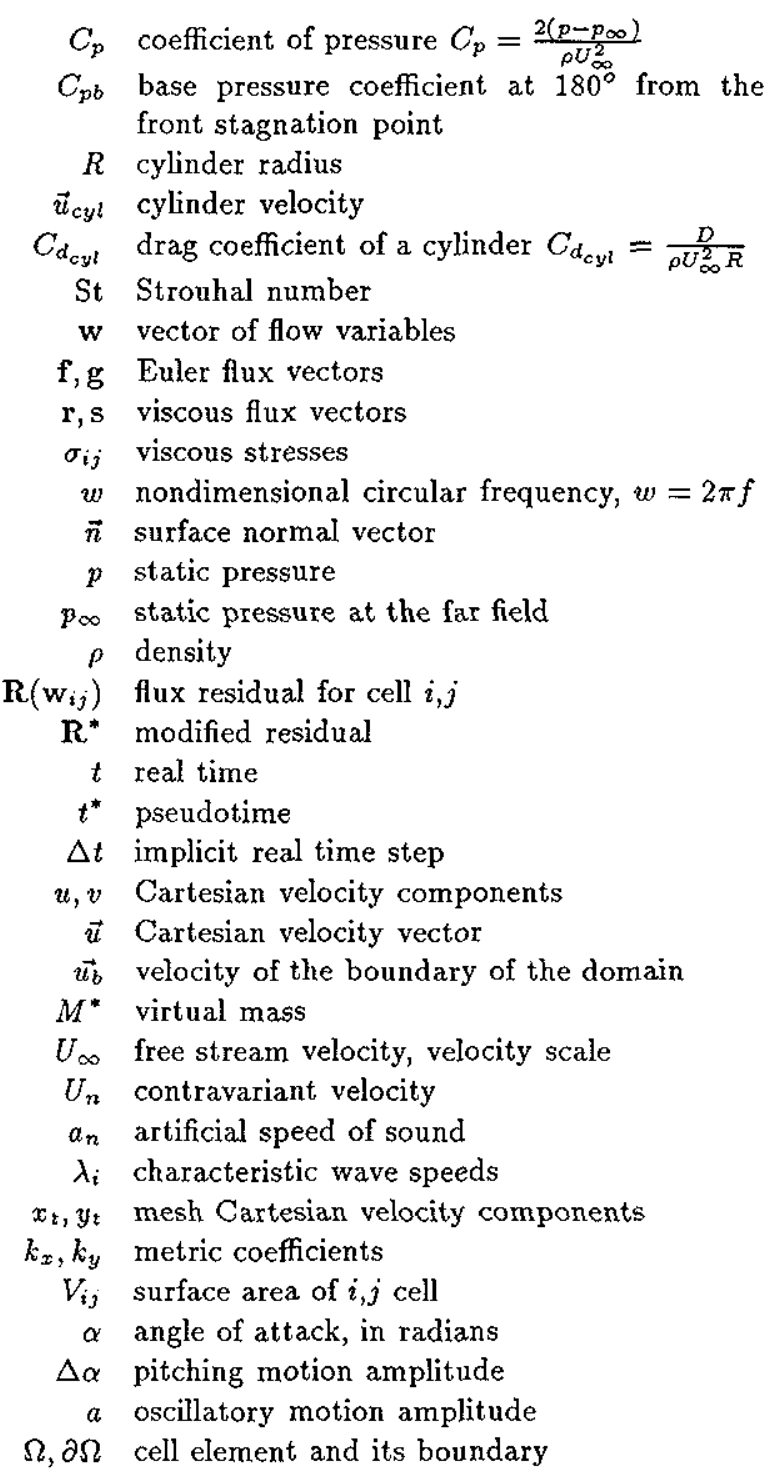

\section{Introduction}

The fast and accurate algorithms for solution of steady state Euler and Navier-Stokes equations developed in recent years have produced very efficient tools for analysis of stationary flows in complex 
aerospace configurations. In particular, the development of a multigrid schemes has greatly enhanced efficiency of both compressible and incompressible algorithms enabling converged solutions of the threedimensional Euler equations to be obtained in 20 to 50 multigrid steps. However, the increase in convergence rate is achieved at the price of a loss of timeaccuracy. At the same time, efficient time-accurate algorithms for unsteady inviscid and viscous flows are of a great practical interest for a wide range of engineering applications, including free surface, aeroelasticity, and wake flow problems.

In this paper we develop a novel approach to increase efficiency of time dependent calculations of incompressible flows in the primitive variable formulation. The method is presented and validated for the full non-linear Euler as well as Navier-Stokes equations.

One of the major difficulties encountered in the calculation of incompressible flow is the enforcement of the time-independent constraint on the velocity field imposed by the continuity equation. In order to couple the velocity and pressure fields, Chorin's artificial compressibility approach [3] can be employed. The pseudotemporal evolution for pressure renders the system hyperbolic and allows for the application of fast compressible flow algorithms to march toward the steady state. This method has received a lot of attention and has been applied to rotational inviscid flows by Rizzi and Eriksson [4], Dreyer [5] has applied it to low speed two-dimensional airfoils, and Farmer et al $[6,7]$ successfully applied it to steady flows over ship hull forms with the free surfaces. A number of preconditioners have been proposed and validated for steady flow calculations [5]. However, these approaches sacrifice time accuracy. Time-accuracy can be recovered by adding the discretized temporal momentum derivatives to the spatial residuals at each mesh point and driving those to zero on each time step, as was proposed by Kwak et al [8] for incompressible flows. This strategy has also been used by Miyake et al. [9] using an explicit, up to a second order accurate discretization in time, and a rational Runge-Kutta scheme for the subiterations.

A second order A-stable discretization of the unsteady terms adopted in the present algorithm allows the stability restriction on the time step to be relaxed by making use of the information coming from the two previous time levels. The resulting system of ordinary differential equations is solved at each time step to drive the augmented residuals to zero. Since the details of the pseudotransient evolution are immaterial, the parameters of the five-stage Runge-Kutta type scheme employed are optimized to speed up the convergence to a stationary state.
The unsteady terms are treated implicitly, as it was originally proposed in [10] for compressible flow calculations, to improve robustness of the algorithm for the case of stiff systems of equations. The key to the algorithm's efficiency is a finite-volume multigrid acceleration technique, developed by Jameson [1], applied to achieve fast convergence in pseudotime.

\section{Governing Equations}

The governing equations of the flow considered are the nonlinear time-dependent incompressible Navier-Stokes equations of the laminar, constant viscosity flow without body forces.

Let $p, u, v$ denote the pressure and Cartesian velocity components respectively. Consider a control volume $\Omega$ with boundary $\partial \Omega$ which moves with Cartesian velocity components $x_{t}$ and $y_{t}$. The equations of motion of the fluid can then be written in integral nondimensional form as

$$
\begin{gathered}
\mathbf{I}^{\mathbf{m}} \cdot \frac{d}{d t} \iint_{\Omega} \mathbf{w} d x d y+\oint_{\partial \Omega}(\mathbf{f} d y-\mathbf{g} d x) \\
=\oint_{\partial \Omega}(\mathbf{r} d y-\mathbf{s} d x),
\end{gathered}
$$

where $\mathbf{w}$ is the vector of flow variables

$$
\mathbf{w}=\left\{\begin{array}{c}
p \\
u \\
v
\end{array}\right\}
$$

the $\mathbf{f}, \mathbf{g}$ are the Euler flux vectors

$$
\mathbf{f}=\left\{\begin{array}{l}
\left(u-x_{t}\right) \\
u\left(u-x_{t}\right)+p \\
v\left(u-x_{t}\right)
\end{array}\right\}, \mathrm{g}=\left\{\begin{array}{l}
\left(v-y_{t}\right) \\
u\left(v-y_{t}\right) \\
v\left(v-y_{t}\right)+p
\end{array}\right\} .
$$

the $\mathbf{r}, \mathbf{s}$ are the viscous flux vectors

$$
\mathbf{r}=\left\{\begin{array}{c}
0 \\
\sigma_{x x} \\
\sigma_{x y}
\end{array}\right\}, \mathbf{s}=\left\{\begin{array}{c}
0 \\
\sigma_{x y} \\
\sigma_{y y}
\end{array}\right\}
$$

where

$$
\begin{aligned}
& \sigma_{x x}=\frac{1}{R e} \cdot \quad\left[2 u_{x}-\frac{2}{3}\left(u_{x}+v_{y}\right)\right] \\
& \sigma_{y y}=\frac{1}{R e} \cdot\left[2 v_{y}-\frac{2}{3}\left(u_{x}+v_{y}\right)\right] \\
& \sigma_{x y}=\frac{1}{R e} \cdot\left[u_{y}+v_{x}\right]
\end{aligned}
$$

where $R e=\frac{U_{\infty} L}{\nu}$ is the Reynolds number, $\nu$ is the kinematic viscosity, $U_{\infty}, L$ are the velocity and the 
length scales of the problem, and the $\mathbf{I}^{\mathbf{m}}$ is the modified identity matrix annihilating temporal derivative of pressure from the continuity equation

$$
\mathbf{I}^{\mathbf{m}}=\operatorname{diag}[0,1,1] \text {. }
$$

$A$ cell-centered formulation is employed in the present method so that, when applied independently to each cell in the mesh domain, Equation 1 results in a set of ordinary differential equations of the form

$$
\frac{d}{d t}\left(\mathbf{w}_{i j} V_{i j}\right)+\mathbf{R}\left(\mathbf{w}_{i j}\right)=0,
$$

where $V_{i j}$ is the surface area of the $i, j$ cell and the residual $\mathbf{R}\left(\mathbf{w}_{i j}\right)$ is obtained by evaluating the sum of the inviscid and viscous flux integrals. The details of the discretization of the viscous fluxes are described in [11]. The convective fluxes are approximated with central differences in space, and a third order artificial dissipation term in conservation form is added in order to prevent the odd-even decoupling as described in[12].

Equation 2 can be approximated implicitly as follows (subscripts $i, j$ are dropped for clarity)

$$
\frac{d}{d t}\left[\mathbf{w}^{n+1} V^{n+1}\right]+\mathbf{R}\left(\mathbf{w}^{n+1}\right)=\mathbf{0},
$$

where the superscript $n+1$ denotes the current time level $(n+1) \Delta t$. If the $\frac{d}{d t}$ operator is approximated by a second order accurate implicit backwards difference formula, Equation 3 becomes

$$
\begin{gathered}
\frac{3}{2 \Delta t}\left[\mathbf{w}^{n+1} V^{n+1}\right]-\frac{2}{\Delta t}\left[\mathbf{w}^{n} V^{n}\right]+\frac{1}{2 \Delta t}\left[\mathbf{w}^{n-1} V^{n-1}\right] \\
+\mathbf{R}\left(\mathbf{w}^{n+1}\right)=0 .
\end{gathered}
$$

The O-mesh used in this work is rigidly attached to the body and does not deform in course of calculation, i.e. no regridding is necessary; the grid is rotated and/or translated only. Note, therefore, that the cell surface area remain constant at all times and can be factored out. The time discretization in Equation 4 is A-Stable [13].

It is convenient at this point to define the modified residual $\mathbf{R}^{*}(\mathbf{w})$ as

$$
\begin{aligned}
\mathbf{R}^{*}(\mathbf{w}) & =\frac{3}{2 \Delta t}\left[\mathbf{w} V^{n+1}\right] \\
- & \frac{2}{\Delta t}\left[\mathbf{w}^{n} V^{n}\right]+\frac{1}{2 \Delta t}\left[\mathbf{w}^{n-1} V^{n-1}\right]+\mathbf{R}(\mathbf{w}),
\end{aligned}
$$

which is to be driven to zero at each mesh point $i, j$, at each time step. This can be accomplished by marching toward a steady state with an explicit multistage scheme applied in the fictitious time ( $p s e u$ dotime) $t^{*}$, using point-implicit discretization of the unsteady source term as in [10].

\subsection{Local Preconditioning}

In the limit of incompressible flow the sound waves travel infinitely fast in all directions and dominate the flow. The disparity in the sound and convective wave speeds makes the governing system of equations ill-conditioned. In order to render the system of equations more suitable for numerical computation, a local preconditioning matrix $\mathbf{P r}$ is introduced yielding

$$
\frac{d \mathbf{w}}{d t^{*}}+\mathbf{P r} \cdot \mathbf{R}^{*}(\mathbf{w})=0,
$$

where

$$
\operatorname{Pr}=\operatorname{diag}\left[\Gamma^{2}, 1,1\right] .
$$

The parameter $\Gamma$ is selected to improve the rate of convergence in pseudotime. Since the unsteady terms enter the modified residual as the source terms, and do not affect the range of the wave speeds present in the system 5 , the form suggested by Rizzi and Eriksson [4], and later by Dreyer [5] can be used. It is adopted in the following form

$$
\Gamma^{2}=\max \left(C_{1}, C_{2}\left(u^{2}+v^{2}\right)\right),
$$

where $C_{1}=0.25$ and $C_{2}=1$.

Equation 5 represents an explicit set of ordinary differential equations to be solved on each time step. It is easy to see, that once the steady state in pseudotime $t^{*}$ is achieved, the modified residuals $\mathbf{R}^{*}(\mathbf{w})$ are driven to zero at each mesh point, yielding the solution of Equation 4 at the new, $n+1$ time level. Then, the time levels in Equation 4 are shifted by minus one, and the new steady state problem is set up. Thus, the original unsteady problem 2 is converted into a number of steady state calculations in $t^{*}$. A five-stage Runge-Kutta type scheme is used to advance the solution in pseudotime with the implicit calculation of the unsteady terms as in [10] and, since the details of the pseudotransient evolution are immaterial, the parameters of the scheme are optimized for faster convergence. In addition, other standard convergence acceleration techniques like the local pseudotime stepping and residual averaging are applied following [5].

A very efficient multigrid strategy is employed to achieve fast convergence on each time step. The details of the multigrid scheme used can be found in [1].

\subsection{Boundary Conditions}

For viscous calculations, a no-slip condition is imposed on the solid boundary by setting the flow velocity equal to that of the body, and a zero pressure gradient normal to the surface is specified to compute the pressure. The boundary conditions used for the Euler calculations are described in [14]. 
Following the guidelines given in [15], an approximate non-reflecting boundary conditions based on the linearized characteristics approach are constructed to improve the rate of convergence in pseudotime. The linearized characteristic problem is solved along the direction $\vec{n}$, normal to the boundary of the computational domain, each time the flow variables are being updated. The appropriate Jacobian matrix and the left and right eigenvectors, corresponding to the pseudocompressibility approach on a moving mesh, can be found in [8]. If we define the scaled contravariant mesh and flow velocities as

$$
U_{n}=k_{x} \cdot u+k_{y} \cdot v
$$

and

$$
u_{m e s h}=k_{x} \cdot u_{b}+k_{y} \cdot v_{b},
$$

where the $k_{x}, k_{y}$ are the metric coefficients, then the corresponding spectrum of eigenvalues is given by

$$
\begin{aligned}
& \lambda_{1}=U_{n}+u_{m e s h} \\
& \lambda_{2}=\left(U_{n}+\frac{1}{2} u_{m e s h}\right)+a_{n} \\
& \lambda_{3}=\left(U_{n}+\frac{1}{2} u_{m e s h}\right)-a_{n}
\end{aligned}
$$

The artificial speed of sound $a_{n}$ is

$$
a_{n}=\sqrt{\left(U_{n}+\frac{1}{2} \cdot u_{m e s h}\right)^{2}+\Gamma^{2} \cdot\left(k_{x}^{2}+k_{y}^{2}\right)} .
$$

A single level halo of fictitious cells is added to the grid, and the corresponding far field values are taken to be equal to the free stream values. In the practical implementation, the similarity transform of the Jacobian matrix [8] is computed on the outer boundary of the domain at each stage of the RungeKutta scheme. Then, depending on the sign of the corresponding wave speed (Equation 6), the characteristic variables in the dummy cells are either interpolated from the interior of the domain, or computed from the free stream values. Finally, the fluxes on the far field boundary are calculated using the velocity and pressure values in the fictitious cells, recovered using the inverse similarity transform [8].

\section{Results and Discussion}

An extensive validation of the basic algorithm with multigrid has been presented in [14] for both timedependent and stationary inviscid flows. Also, timeaccurate Euler calculations on unstructured grids have been presented in [16]. In this work the algorithm is extended to include laminar viscous stress terms. It is also modified by using a pointimplicit formulation in pseudotime for the unsteady terms, and by the introduction of approximate nonreflecting far field boundary conditions. In order to validate the flow solver further, both viscous and inviscid time-dependent problems are considered and discussed next.

\subsection{Oscillating Circular Cylinder}

The inviscid incompressible flow over a sinusoidally oscillating circular cylinder is considered first. This problem has an analytic solution and provides a severe test for the computational algorithm since no physical dissipation is present. The diameter of a cylinder and a period of forced oscillations are chosen to be the length and the time scales of the problem, so that the nondimensional frequency $f=1$ results. The coordinate of the center of a cylinder is set to

$$
x_{\mathrm{cyl}}(t)=a \cdot \sin (w t-\varphi),
$$

where the motion amplitude $a$ is equal to 0.1 and the initial phase $\varphi$ is $90^{\circ}$. The fluid velocity and the static pressure at infinity $P_{\infty}$ are assumed to be zero. The exact solution for the incompressible inviscid flow over a moving cylinder can be found in [17]. The velocity field and the surface pressure distribution reduce in this case to

$$
\begin{aligned}
& u(\theta, t, r)=\frac{0.25}{r^{2}} \cdot u_{c y l}(t) \cdot \cos 2 \theta \\
& v(\theta, t, r)=\frac{-0.25}{r^{2}} \cdot u_{c y l}(t) \cdot \sin 2 \theta
\end{aligned}
$$

$P_{s}(\theta, t)=P_{\infty}+u_{c y l}^{2}(t) \cdot\left(2 \cos ^{2} \theta-1.5\right)+\frac{d u_{c y l}(t)}{2 d t} \cdot \cos \theta$ where $r$ is the distance to the center of a cylinder and $\theta$ is measured clockwise from the direction of the $x$-axis. The third term in the pressure equation produces a nonzero net contribution to the dynamic drag force acting on a cylinder. The resulting drag coefficient due to the apparent mass effect is

$$
C_{d_{c y l} l}(t)=\frac{a w^{2} \pi}{2} \cdot \sin (w t-\varphi) .
$$

A grid refinement study is carried out using three $\mathrm{O}$-meshes rigidly attached to the cylinder consisting of $65 \times 33,129 \times 65$ and $257 \times 129$ grid points respectively. The cylinder is initially at rest with $x_{\text {cyI }}(0)=-a$. The time step is $\Delta t=0.025$ and the far field boundary is placed 16 diameters away from the center of the cylinder. On each grid, the maximum modified residual in the divergence equation, given by $\frac{1}{\Gamma^{2}} \frac{\delta P}{\delta t^{*}}$, is reduced below $10^{-4}$ on each time step using approximately 50 multigrid $\mathrm{W}$-cycles in pseudotime. The evolution of the drag coefficient computed on the $65 \times 33$ grid is compared in Figure 1 with the exact solution (Equation 8), which is shown using a solid line. The calculation on the coarsest grid is found to overpredict the maximum drag amplitude by approximately $1.7 \%$, while the results for the two finer grids coincide with the solid line. The order of convergence in the grid refinement study can be estimated by the logarithm of the ratio of the computed drag amplitudes

$$
\log _{2}\left(\frac{\max \left(C d_{65 \times 33}\right)-\max \left(C d_{129 \times 65}\right)}{\max \left(C d_{129 \times 65}\right)-\max \left(C d_{257 \times 129}\right)}\right)=1.84,
$$


which is close to 2 . This is indeed consistent with the second order accuracy of the spatial discretization which was used. The recovered inviscid pressure and velocity component distributions on the surface of the cylinder, given by Equations 7, are shown in Figures 2, 3 and 4 .

\subsection{Parallel Vortex Shedding from a Circular Cylinder}

The unsteady laminar vortex shedding from a circular cylinder for $R e \leq 200$, based on the cylinder diameter and a free stream velocity, was selected as the second test problem. The detail of the $257 \times 257$ O-mesh used is shown in Figure 5. The normal spacing of the grid next to the surface is 0.0003 diameters, and a relatively fine resolution of approximately 0.1 diameter is maintained in the radial direction up to the far field boundary. The far field boundary is placed at a distance of 16 diameters from the center of the cylinder. The focus of this study is on the mean features of the asymptotic wake behavior. Thereforc, in order to speed up the transition to the limiting cycle and save the CPU time, the cylinder is initially rotated about it's center with the amplitude of $2^{\circ}$ and then stopped. The initial motion with different amplitudes seem to influence the onset time of the asymmetric shedding, but not the characteristics of the limiting cycle itself.

The computed time evolution of the coefficients of drag and lift for the flow with $R e=150$ is shown in Figure 6. The converged Strouhal frequency was found to be equal $S t=0.182$, while the values of the aerodynamic coefficients, corresponding to a limiting cycle, are: $C l=0.000 \pm 0.486$ and $C d=1.168 \pm 0.025$. A typical convergence history for a pseudotransient calculation performed on each time step is plotted in Figure 7. Once the asymmetric shedding developed, 15 multigrid $\mathrm{W}$-cycles using seven grid levels were sufficient to enforce the divergence free constraint in each cell better than $10^{-5}$, as measured by the maximum continuity residual, as can be seen in Figure 7. The maximum and the root mean squared residuals in the continuity equation, obtained after 15 subiterations in pseudotime, are plotted for each time step in Figure 8. The net numerical mass flux out of the computational domain

$$
\frac{d M^{*}}{d t}=\oint_{\partial \Omega}\left(\vec{u}-\overrightarrow{u_{b}}\right) \cdot \vec{n} d l,
$$

indicates the amount of the "virtual mass" accumulated during any particular time step and, in the conservative formulation, provides an additional, global convergence criteria for the deviation of the computed velocity from the solenoidal field. The evolution of this quantity during a typical pseudotransient calculation is also shown in Figure 7. In order to assess the effects of the spatial discretization, the two auxiliary calculations with the $R e=150$ are performed on two successively coarser grids with, respectively, $129 \times 129$, and $65 \times 65$ grid points. The coarser grids are constructed by eliminating the alternate points from the finest $256 \times 256$ grid, resulting in a somewhat lower resolution in the boundary layer. The time histories of the drag and lift coefficients for all three grids are presented in Figure 9. The order of convergence can be estimated using the ratios of the amplitudes of the oscillations of the aerodynamic coefficients as well as the Strouhal numbers computed on the three grids

$$
\begin{gathered}
\log _{2}\left(\frac{\mathrm{St}_{65 \times 65}}{\mathrm{St}_{129 \times 129}-\mathrm{St}_{129 \times 129}}\right)=2.27, \\
\log _{2}\left(\frac{\max \left(C l_{65 \times 65}\right)}{\max \left(C l_{129 \times 129}\right)-\max \left(C l_{129 \times 129}\right)}\right)=1.83, \\
\log _{2}\left(\frac{\max \left(C d_{65 \times 65}\right)}{\max \left(C d_{129 \times 129}\right)-\max \left(C d_{129 \times 129}\right)}\right)=2.01,
\end{gathered}
$$

which are indeed close to 2 . This is consistent with the second order accuracy of the spatial discretization and also in agreement with the value obtained for the inviscid calculations above. A more detailed grid refinement study using grids even finer then $256 \times 256$ is planned following the parallelization of the present solver [18].

The instantaneous distribution of the vorticity in the entire computed wake is shown in Figure 10. It can be seen that no spurious reflections are present at the exit boundary.

Figure 11 shows the instantaneous streamlines computed at different stages of the shedding cycle. The second and the fourth stage shown in Figure 11 correspond approximately to the minimum and the maximum lift which are produced by the low pressure region associated with the attached vortex. The downstream convection of the vortex, as well as the formation of the new vortices, either on the top or on the bottom backward facing parts of a cylinder, correspond to the alternate mirror-image phases of the flow with minimal drag and zero lift. Thus the drag varies with double frequency compared to that of the lift. Qualitatively similar streamline patterns have been presented and discussed in [8] for the flow with $R e=200$.

The laminar wake flow with $R e=200$ has been considered by many authors as a test case, and some of the relevant data, quoted from $[8,9,19]$, are presented in Table 1. However, according to [20], threedimensional vortex shedding occurs at this Reynolds number. Also, the results in [21] suggest that the continuation of the "universal curve" corresponding to the parallel shedding (see the analysis below) and represented by the last entry in Table 1, might provide a better basis for validation of two-dimensional computations, then the actual experimental data for 


\begin{tabular}{|l|l|l|l|}
\hline Reference & Cl & \multicolumn{1}{c|}{ Cd } & St \\
\hline Present & \pm 0.64 & $1.19 \pm 0.042$ & .193 \\
\hline Rogers, Kwak & \pm 0.65 & $1.23 \pm 0.05$ & .185 \\
\hline Miyake et al. & \pm 0.67 & $1.34 \pm 0.043$ & .196 \\
\hline Lecointe et al. & \pm 0.5 & $1.58 \pm 0.0035$ & .194 \\
\hline Lin et al. & & 1.17 & \\
\hline Henderson & & & .197 \\
\hline Kovaznay (exp.) & & & .19 \\
\hline Roshko (exp.) & & & .19 \\
\hline Roshko (fit.) & & & .190 \\
\hline Williamson (fit.) & & & .197 \\
\hline
\end{tabular}

Table 1: Flow over a circular cylinder at Reynolds number 200: coefficients of lift and drag and Strouhal number.

this particular Reynolds number. Taking into account those considerations, and especially in view of the wide scattering of the data in Table 1 , it appears that a lower Reynolds number flow $(R e=150$ for example) would provide a more meaningful validation of the two-dimensional numerical computations.

Recent experimental studies of the wake of circular cylinders $[22,20]$ showed that a parallel shedding can be observed in the range of $49<R e<180$, provided that special care is taken to control the end effects. Above $R e=180$, three-dimensional shedding modes are observed, while for Reynolds numbers below 49 the wake is stationary. A continuous relation $S t(R e)$ of the form

$$
S t(R e)=\frac{A}{R e}+B+C \cdot R e,
$$

where $A, B$ and $C$ are constants, was proposed by Williamson [23] to describe the parallel vortex shedding believed to be accurate within $1 \%$. Also, as originally proposed by G.Brown and shown in [23], the experimental data for the three-dimensional oblique shedding in the same range of $R e$ numbers do collapse on the same "universal curve" 10 , provided that the Strouhal number is scaled with the inverse cosine of the shedding angle. These findings suggest that a comparison of numerical results with the empirical relation 10 would provide a particularly meaningful validation of any numerical method. Such a study has been recently carried out by R.Henderson [21] using an unstructured spectral element method.

The computed $S t(R e)$ data for the set of calculations in the range of $R e \leq 200$ are compared with the experimental curves and other computations $[23,21,24,22]$ in Figure 12. The curve fitting coefficients, corresponding to Equation 10, which were used for the comparison, are summarized in Table 2.

The experimental data of Williamson [23] are shown in Figure 12 for the range $49<R e<180$ by

\begin{tabular}{|l|c|l|l|r|}
\hline Source & Quantity & \multicolumn{1}{|c|}{$A$} & $B$ & $C \cdot 10^{4}$ \\
\hline$[23]$ & St & -3.3265 & .1816 & 1.600 \\
\hline$[24]$ & St & -4.50 & .212 & - \\
\hline$[22]$ & St & -5.35 & .212 & - \\
\hline$[21]$ & St & -3.3658 & .1831 & 1.627 \\
\hline$[20]$ & Cpb & -14.3500 & .6950 & 16.920 \\
\hline$[21]$ & Cpb & -10.1090 & .6380 & 20.570 \\
\hline
\end{tabular}

Table 2: The coefficients of the "universal curve" (Equation 10).

a solid line with the ticks indicating an estimated accuracy of $\pm 1 \%$. The present data are found to be in excellent -better than 1\%- agreement with the "universal curve" in this range of Reynolds numbers. Our data are also in good agreement with the experimental curve obtained by Roshko [24] and the unstructured spectral element computations by Henderson [21]. However, more computations may be necessary to define the coefficients of the curve using the present method.

The computed variation of the base pressure coefficient with Reynolds number $C p b(R e)$ is shown in Figure 13. Our data exhibit the same trend as other computational and experimental results [21,24], yet they differ from the latter by $2 \div 3 \%$. The results in Figure 13 suggest that a detailed study of the effects of artificial dissipation on the accuracy of the results might be necessary to determine the exact source of the difference. On the other hand, the experimental data used in Figure 13 for the low Reynolds number regime are generally considered to be somewhat less accurate then the Strouhal number curve fit, Equation 10.

Below $R e<49$, the wake of a circular cylinder is stationary [20]. The computed evolution of the drag and lift coefficients for $R e=30$ is shown in Fig. ure 14. A decaying response is observed in the calculation, further confirming that the present method captures correct the asymptotic behavior of wake flows for the range of Reynolds numbers considered.

\subsection{Vortex Shedding from the NACA0012 Airfoil}

The viscous flow over a NACA0012 airfoil with $R e=1000 .$, based on the chord length and the free stream velocity, fixed at $20^{\circ}$ angle of attack is considered as yet another test problem. This case is used to assess the effects of the truncation of the domain and time accuracy. In order to isolate the effects of the location of the outer boundary, the same core mesh is preserved for all the calculations while the distance of the outer boundary is increased. Four Omeshes are constructed using $129 \times 129,129 \times 193$, $129 \times 257$ and $129 \times 321$ nodes with the far field boundary placed at approximately $6,11,16$ and 21 


\begin{tabular}{|c|c|c|c|}
\hline Far field & $\mathrm{Cl}_{\max , \min }$ & $\mathrm{Cd}_{\max , \min }$ & $\overline{\mathrm{St}}$ \\
\hline$\overline{6 \cdot c}$ & $1.140,0.706$ & $0.444, \quad 0.347$ & .5435 \\
\hline $11 \cdot c$ & $1.138,0.699$ & $0.442, \quad 0.343$ & .5308 \\
\hline $16 \cdot c$ & $1.136,0.698$ & $0.441,0.343$ & .5271 \\
\hline $21 \cdot c$ & $1.138,0.700$ & $0.442,0.343$ & .5272 \\
\hline
\end{tabular}

Table 3: Flow over the NACA0012 airfoil: the domain truncation effects.

chords. The detail of the base $129 \times 129$ grid is shown in Figure 15. The finer grids are constructed by adding a halo of extra points to the base grid, maintaining a constant spacing of approximately 0.075 chords in the radial direction.

The transition to a periodic shedding cycle is accelerated by a single initial pitch of the airfoil between the 20 and 25 degree angles of attack. The time step is $d t=0.05$. The time histories of the aerodynamic coefficients, computed on all four grids, are plotted in Figure 16. The computed mean flow quantities are summarized in Table 3.

The location of the far field boundary at 16 chords from the midchord of the airfoil is found to provide a suitable compromise between accuracy - within a fraction of a percent - and cost of the computation. A more elaborate far field model may be employed to attenuate further the effects of the truncation of the domain. Alternatively, the far field boundary could be extended even further from the airfoil, at the expense of the resolution in the far wake. The finest grid used so far has $257 \times 257$ nodes with an outer boundary extended 16 chords away from the midchord of the airfoil. The calculation with the time step $d t=0.05$ is presented in Figure 17 . Twenty multigrid $\mathrm{W}$-cycles in pseudotime (using 7 grid levels) were sufficient to reduce the maximum continuity residual to the $10^{-5}$ level on each time step during the limiting cycle as shown in Figure 18. The following mean flow quantities were computed: $\mathrm{St}=0.5199, \mathrm{Cl}_{\max }=1.1696, \mathrm{Cl}_{\min }=0.6748$, and $\mathrm{Cd}_{\max }=0.4536, \mathrm{Cd}_{\min }=0.3331$. The computational results agree well, apart from the lower value of the predicted $\mathrm{Cd}_{m i n}$, with those obtained by Miyake et al. [9] using a $128 \times 128$ grid. The method in [9] employs an explicit, up to a second order accurate time discretization scheme, rational RungeKutta discretization in pseudotime and a multigrid convergence acceleration, developed by Jameson [1].

A time resolution study for the base $129 \times 129$ grid was carried out. The results obtained using time steps $d t=.1, .05, .025$ and .0125 are summarized in Table 4, and illustrated in Figure 19. The present algorithm employs an A-stable discretization of the unsteady terms to relax the stability constraint on the time step used and to substantially improve efficiency of the time-dependent calculations [14]. At the same time, the results in Table 4 suggest that

\begin{tabular}{|c|c|c|c|}
\hline$d t$ & $\mathrm{Cl}_{\max , \min }$ & $\mathrm{Cd}_{\max , \min }$ & St \\
\hline 0.1 & $1.114,0.711$ & $0.435, \quad 0.347$ & .5181 \\
\hline 0.05 & $1.140,0.706$ & $0.444,0.347$ & .5435 \\
\hline 0.025 & $1.124,0.686$ & $0.438,0.340$ & .5400 \\
\hline 0.0125 & $1.124,0.683$ & $0.438,0.339$ & .5405 \\
\hline
\end{tabular}

Table 4: Flow over the NACA0012 airfoil: time resolution study.

between 40 and 80 time steps per shedding cycle are required to resolve the mean quantities of the laminar wake of the airfoil with an accuracy better than $.5 \%$. This suggests that, for certain types of flow, the discretization of the unsteady terms with higher order backward difference formulas [25] may provide a better compromise between stability and accuracy at the expense of the extra storage necessary for accommodating the additional time levels. This approach has been successfully employed for compressible flow calculations by Alonso et al. [26] using a similar implicit multigrid driven method, coupled with a third order accurate time discretization. A point-implicit implementation of higher order differencing in time could improve further the efficiency of our present solver.

\section{Conclusions}

A new algorithm which couples the artificial compressibility approach with a very efficient multigrid acceleration technique has been developed. The method employs a fully implicit A-stable time discretization and requires the solution of a pseudosteady state problem at every time step.

This formulation has the advantage that the time step is determined solely by the physical time scales of the problem, while a fast rate of convergence of the iteration is achieved by using multigrid acceleration. The scheme is very efficient and requires less than $10^{-2}$ sec. CPU time per time step, per mesh point on an IBM RISC6000-580 processor.

The accuracy of the scheme is assessed by grid refinement studies and by comparison with published data for several inviscid and viscous test cases. The results obtained compare favorably with available experimental data, as well as numerical results computed by other authors. We conclude that the proposed scheme provides an accurate and efficient way of computing time-dependent incompressible flows.

Extension of the present method to a higher order accurate discretization in time is currently under way. This will allow the time accuracy constraints on the time step to be relaxed and improve even further the efficiency of our method. The new algorithm is highly suitable for parallel computing [18], and a parallelization of the scheme will enable full three-dimensional unsteady viscous calculations. 


\section{Acknowledgement}

We are grateful to Dr. Henderson for providing us with his data.

Support for this work was provided through by the Office of Naval Research through Grant N00014-93I-0079, under the supervision of Dr. E.P. Rood.

\section{References}

[1] Jameson, A., "Solution of the Euler Equations by a Multigrid Method," Applied Mathematics and Computation, Vol. 13, pp. 327-356, 1983.

[2] Jameson, A., "Time Dependent Calculations Using Multigrid, with Applications to Unsteady Flows Past Airfoils and Wings," AIAA Paper 91-1596, June 1991.

[3] Chorin, A.J., "A Numerical Method for Solving Incompressible Viscous Flow Problems," Journal of Computational Physics, Vol. 2, pp. 12-26, 1967.

[4] Rizzi, A. and Eriksson L.-E., "Computation of Inviscid Incompressible Flow with Rotation," Journal of Fluid Mechanics, Vol. 153, pp. 275$312,1985$.

[5] Dreyer, J., "Finite Volume Solutions to the Unsteady Incompressible Euler Equations on Unstructured Triangular Meshes," M.S. Thesis, MAE Dept., Princeton University, 1990.

[6] Farmer, J., Martinelli, L. and Jameson, A., "Fast Multigrid Method for Solving Incompressible Hydrodynamic Problems with Free Surfaces," AIAA Journal, Vol.32, No.6, pp.1175-1182, June 1994.

[7] Farmer, J., Martinelli, L. and Jameson, A., "A Fast Multigrid Method for Solving the Nonlinear Ship Wave Problem with a Free Surface," presented at the 6th International Conference on Numerical Ship Hydrodynamics, Iowa City, August 1993.

[8] Rogers, S.E. and Kwak, D., "Upwind Differencing Scheme for the Time-Accurate Incompressible Navier-Stokes Equations," AIAA Journal, Vol. 28, No. 2, pp. 253-262, February 1990.

[9] Miyake, T., Sakamoto, Y., Tokunaga, H. and Satofuka, N., "Numerical Solution of Incompressible Flow Using Two-Step, One-Stage Runge-Kutta Time Integration Scheme," Presented at the 1st European Computational Fluid Dynamics Conference, Brussels, Belgium, 7-11 September, 1992.
[10] Melson, N.D., Sanetrik, M.D. and Atkins, H.L., "Time Accurate Navier-Stokes calculations with Multigrid Acceleration," presented at the 6th Copper Mountain Conference on Multigrid Methods, Copper Mountain, 4-9 April, 1993.

[11] Martinelli, L., "Calculations of Viscous Flows with a Multigrid Method," Ph.D. Thesis, MAE Dept., Princeton University, October 1987.

[12] Farmer, R.J., "A Finite Volume Multigrid SoIution to the Three Dimensional Nonlinear Ship Wave Problem," Ph.D. Thesis, MAE Dept., Princeton University, January 1993.

[13] Jeltsch, R., "Stability on the Imaginary Axis and A Stability of Linear Multistep Methods," BIT, Vol. 18, pp. 170-174, 1978.

[14] Belov, A., Martinelli, L. and Jameson, A., "A Novel Fully Implicit Multigrid Driven Algorithm for Unsteady Incompressible Flow Calculations," Computational Fluid Dynamics '94, Proceedings of the 2nd European Computational Fluid Dynamics Conference, Stuttgart, Germany, 5-8 September 1994, John Wiley \& Sons, 1994.

[15] Giles, M.B., "Non-Reflecting Boundary Conditions for the Euler Equations," Technical Report TR-88-1, MIT Computational Fluid Dynamics Laboratory, 1988.

[16] Lin, P.T., "Implicit Time Dependent Calculations for Compressible and Incompressible Flows on Unstructured Meshes," M.S.E. Thesis, MAE Dept., Princeton University, November 1994 .

[17] Landau, L.D. and Lifshitz, E.M., "Fluid Mechanics," Pergamon Press, 1987.

[18] Alonso, J.J., Martinelli, L. and Jameson, A., "Multigrid Unsteady Navier-Stokes Calculations with Aeroelastic Applications," AIAA Paper 95-0048, AIAA 33rd Aerospace Sciences Meeting, Reno, Nevada, January 1995.

[19] Henderson, R.D., Private communication.

[20] Williamson, C.H.K. and Roshko, A., "Measurements of base pressure in the wake of a cylinder at low Reynolds numbers," Z.Flugwiss. Weltraumforsch, 14, pp.38-46, 1990.

[21] Henderson, R.D., "Unstructured Spectral Element Methods: Parallel Algorithms and Simulations," Ph.D. Thesis, MAE Dept., Princeton University, June 1994. 
[22] Mammache, M. and Gharib, M., "An experimental study of the parallel and oblique vortex shedding from circular cylinders," J. Fluid Mech., Vol. 232, pp. 567-590, 1991.

[23] Williamson, C.H.K., "Defining a universal and continuous Strouhal-Reynolds number relationship for the laminar vortex shedding of a circular cylinder," Phys. Fluids, Vol. 31, pp. 2742$2744,1988$.

[24] Roshko, A., "On the development of turbulent wakes from vortex streets,"NACA Rep.1191,1954.

[25] Gear, C.V., "Numerical Initial value Problems in Ordinary Differential Equations," Prentice Fall, Englewood Cliffs, New Jersey, 1971.

[26] Alonso, J.J. and Jameson, A., "Fully-Implicit Time-Marching Aeroelastic Solutions," AIAA Paper 94-0056, January 1994.

[27] Lecointe, Y. and Piquet, J., "On the Use of Several Compact Methods for the Study of Unsteady Incompressible Viscous Flow Round a Circular Cylinder," Computers and Fluids, Vol.12, No.4, pp.255-280, 1984.

[28] Lin, C.L., Pepper, D.W., and Lee, S.C., "Numerical Methods for Separated Flow Solutions Around a Circular Cylinder," AIAA Journal, Vol.14, pp.900-907, July 1976.

[29] Kovasznay, L.S.G., "Hot-wire Investigation of the Wake Behind Cylinders at Low Reynolds Numbers," Proceedings of the Royal Society of London, Series A, Vol.198, No.1053, pp.174190, August 1949. 


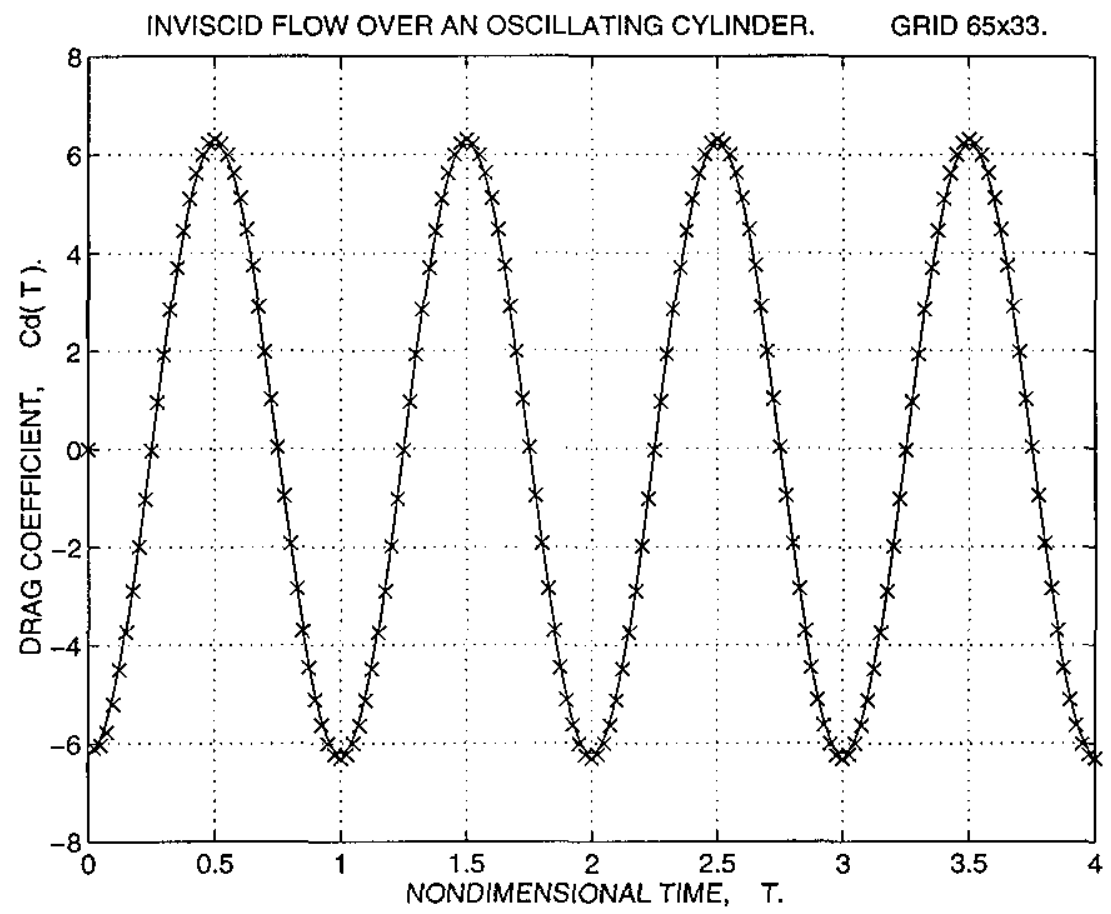

Figure 1: Drag Coefficient Evolution of the Oscillating Cylinder.

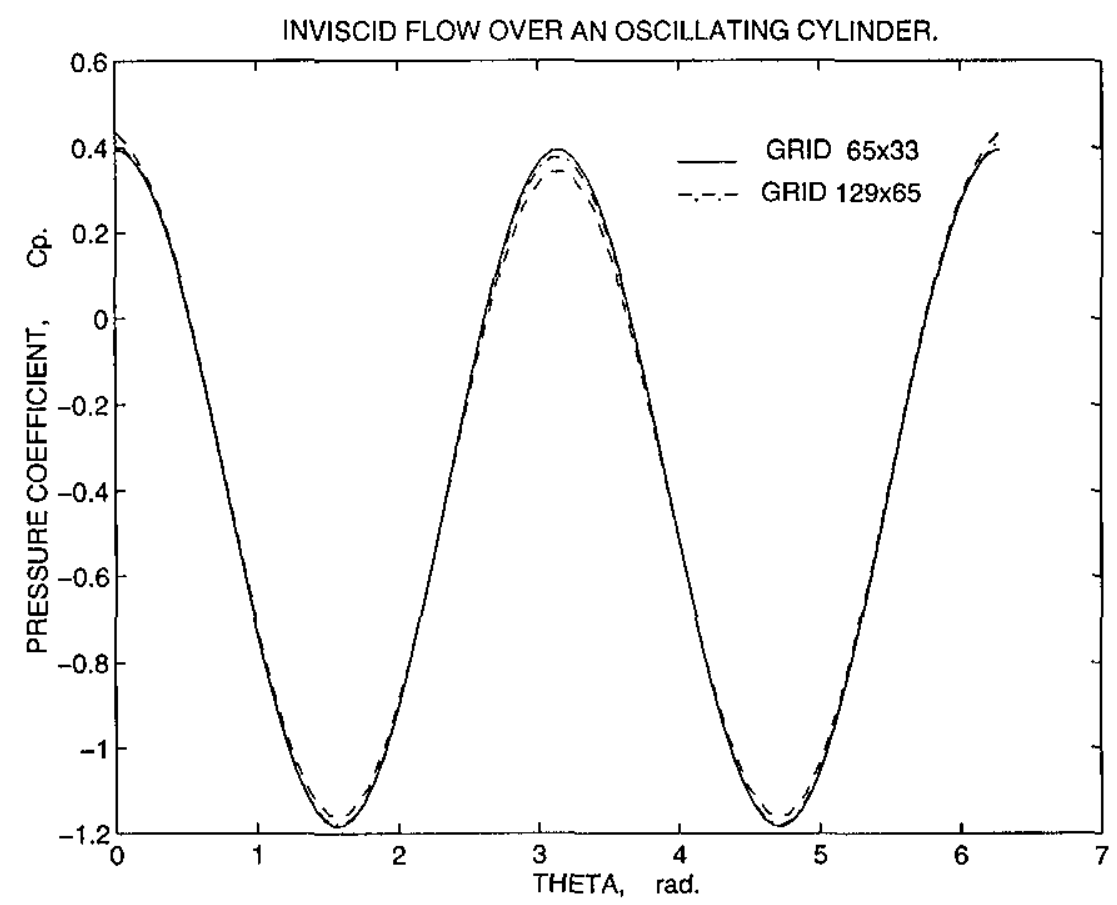

Figure 2: Pressure Coefficient Distribution over the Surface of the Oscillating Cylinder, $t=4.25$. 


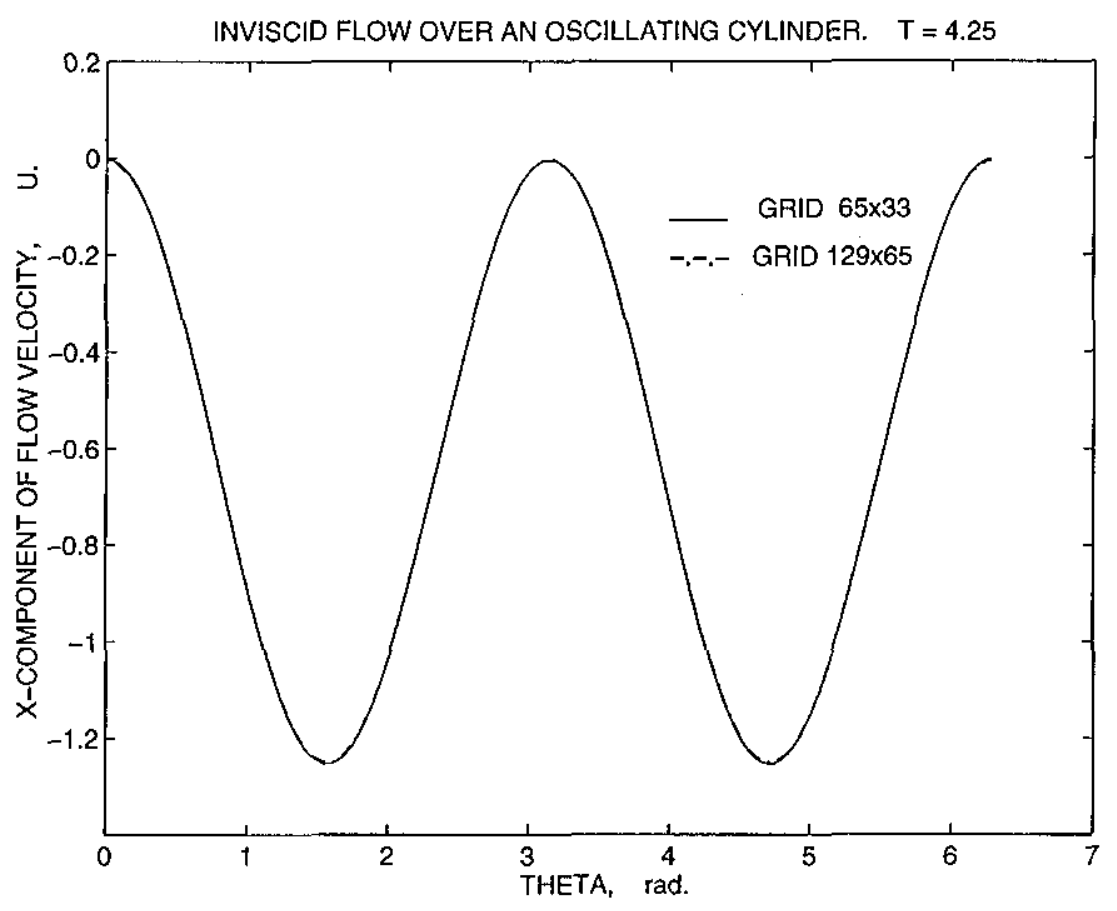

Figure 3: Distribution of the x-Component of Velocity.

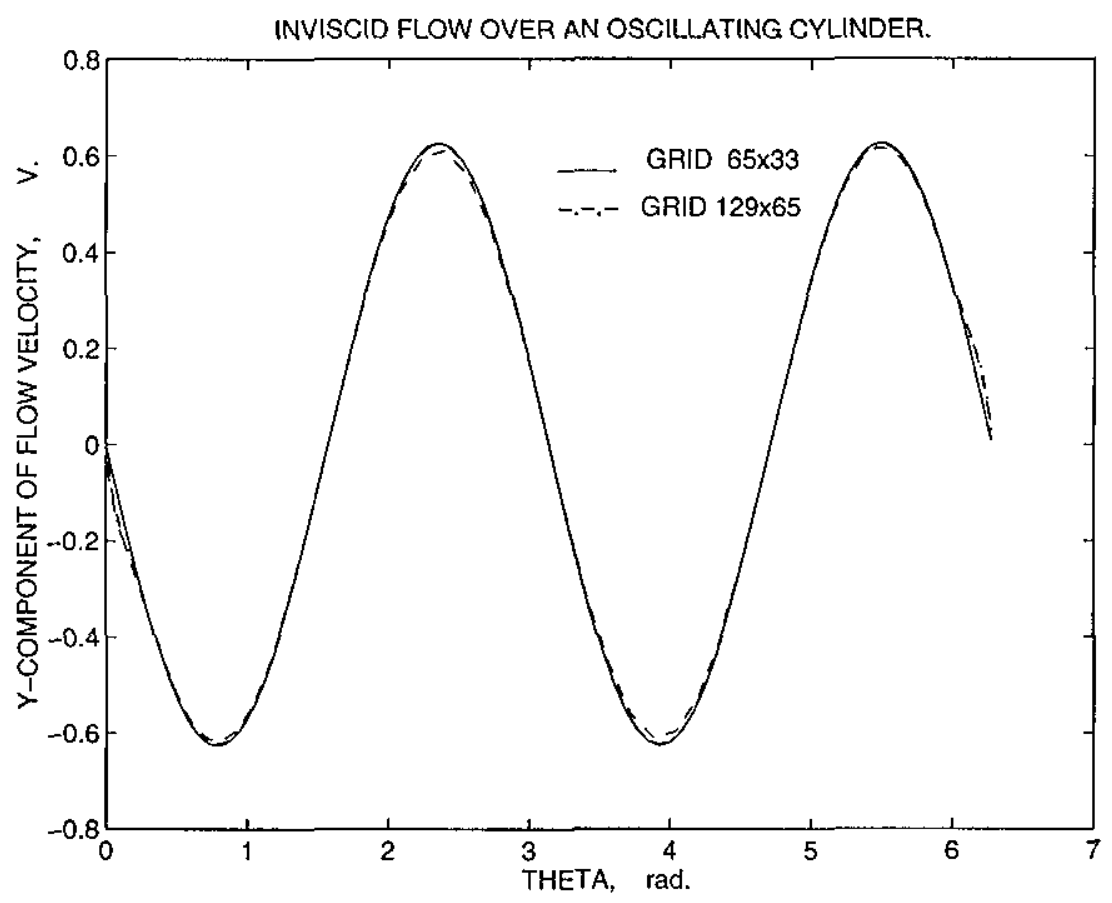

Figure 4: Distribution of the y-Component of Velocity. 


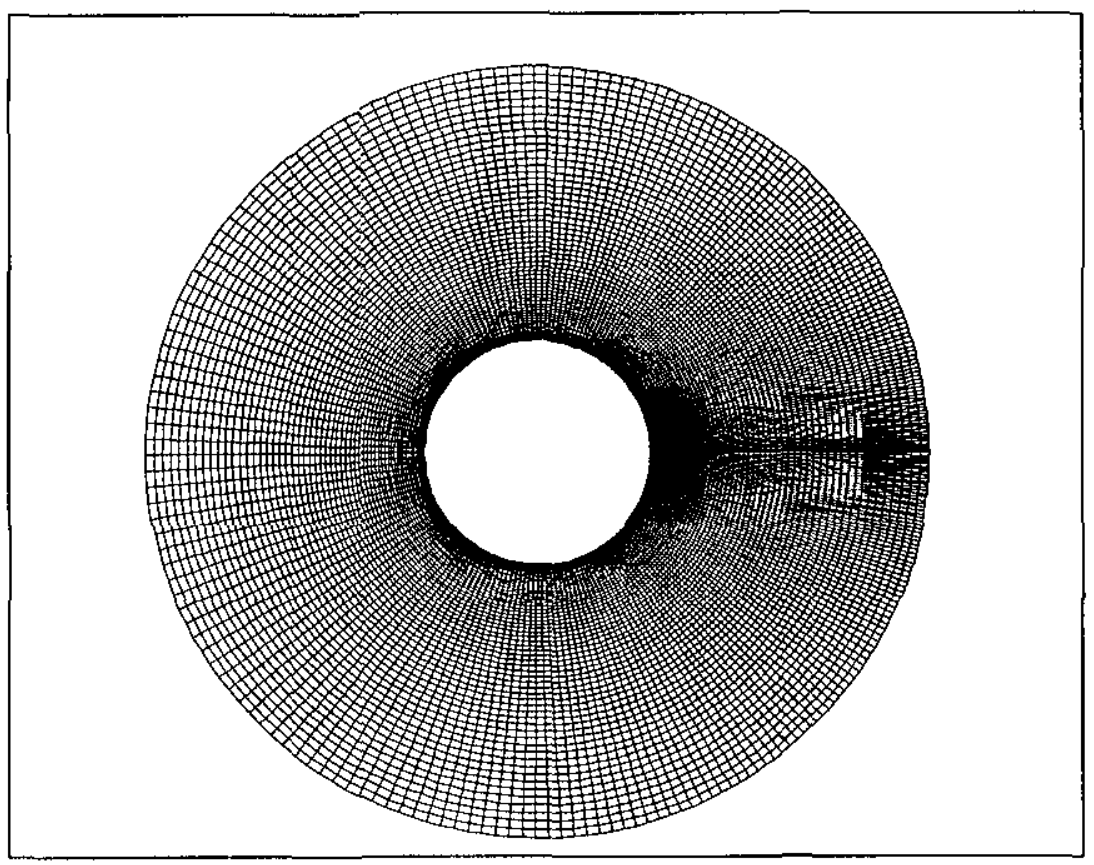

Figure 5: Detail of an O-Mesh.

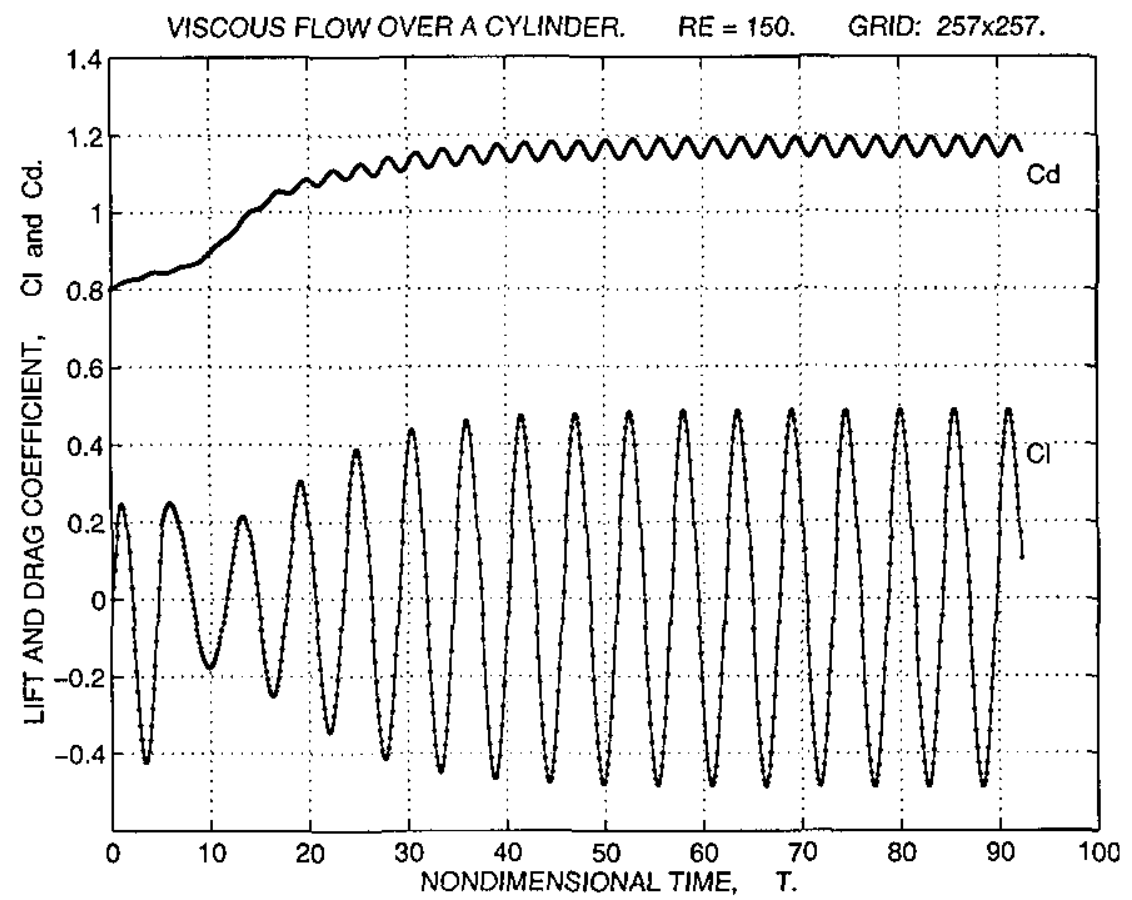

Figure 6: Evolution of the Drag and Lift Coefficients of a Circular Cylnder. 


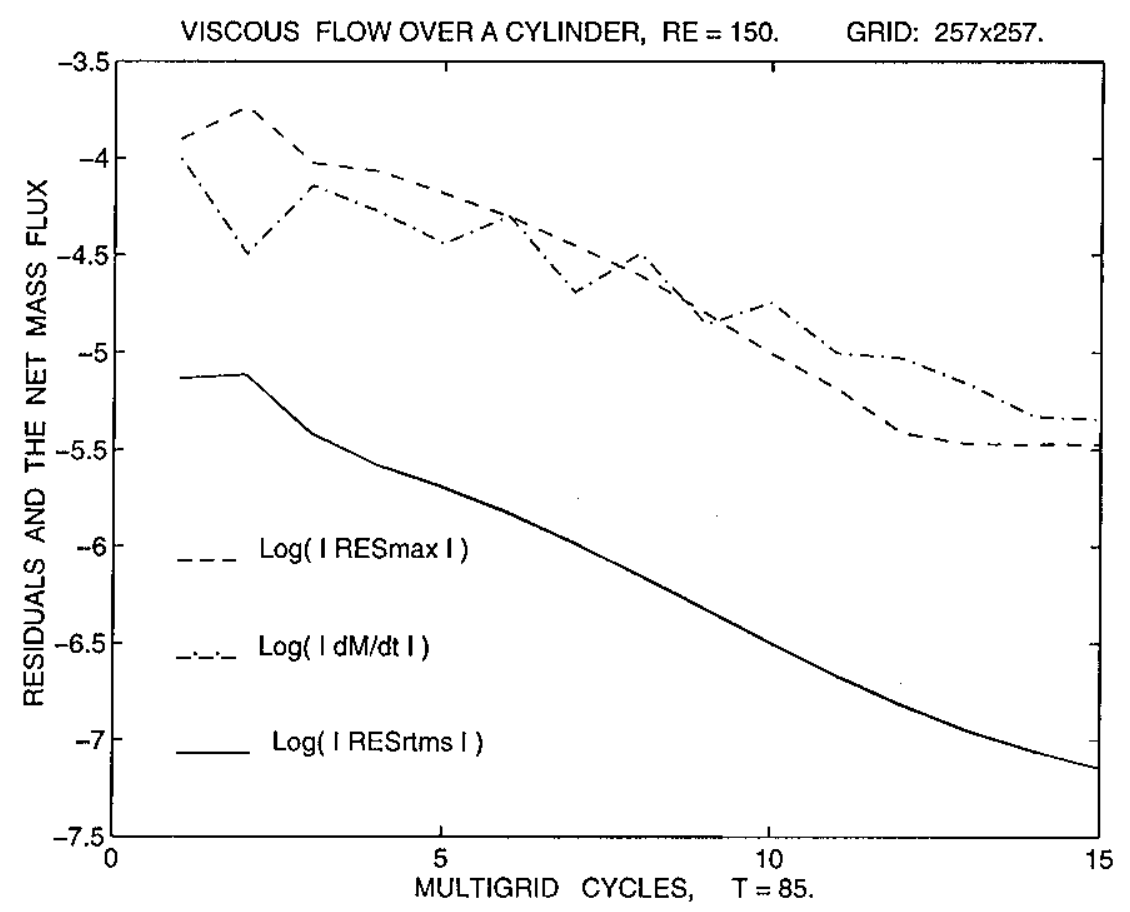

Figure 7: Convergence History as Measured by the Maximum, the Root Mean Squared Continuity Residuals, and by the Net Virtual Mass Flux out of the Domain.

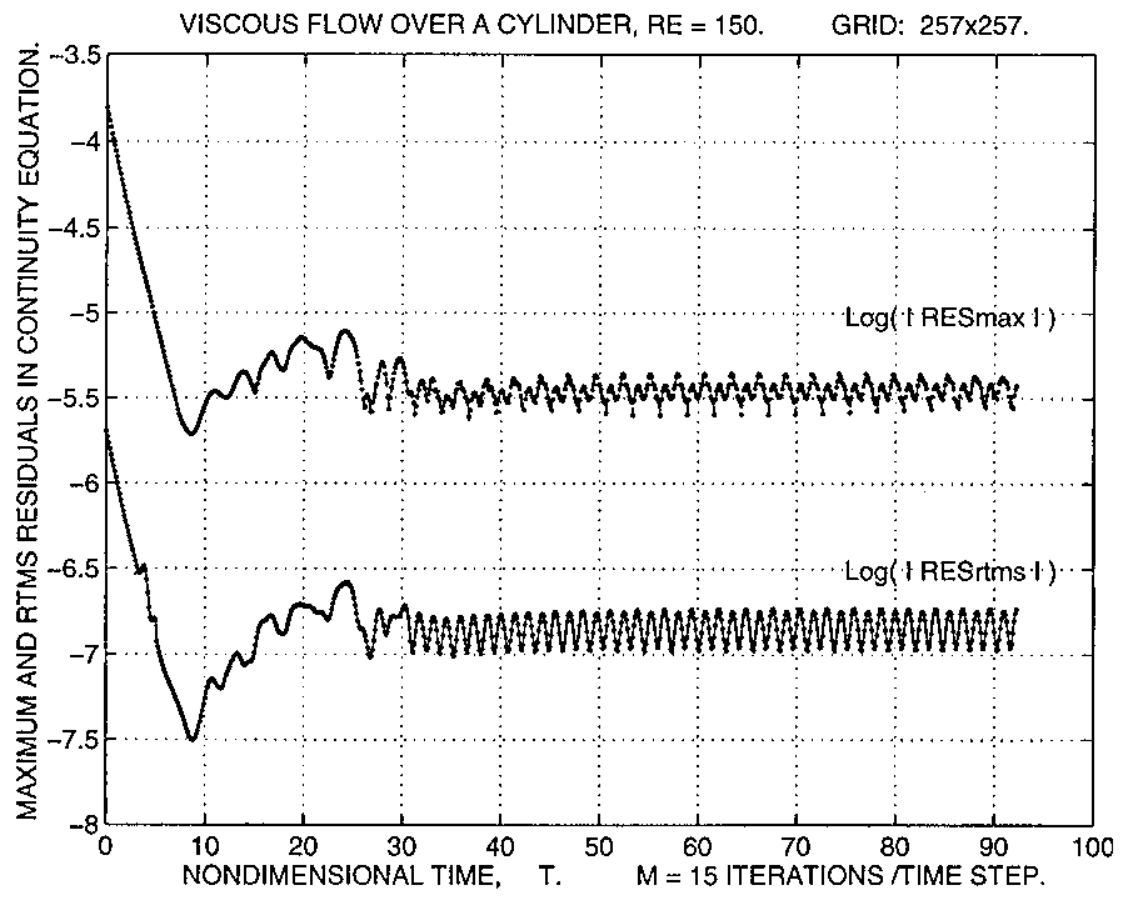

Figure 8: Continuity Residuals Resulted on Each Time Step after 15 Multigrid Cycles. 


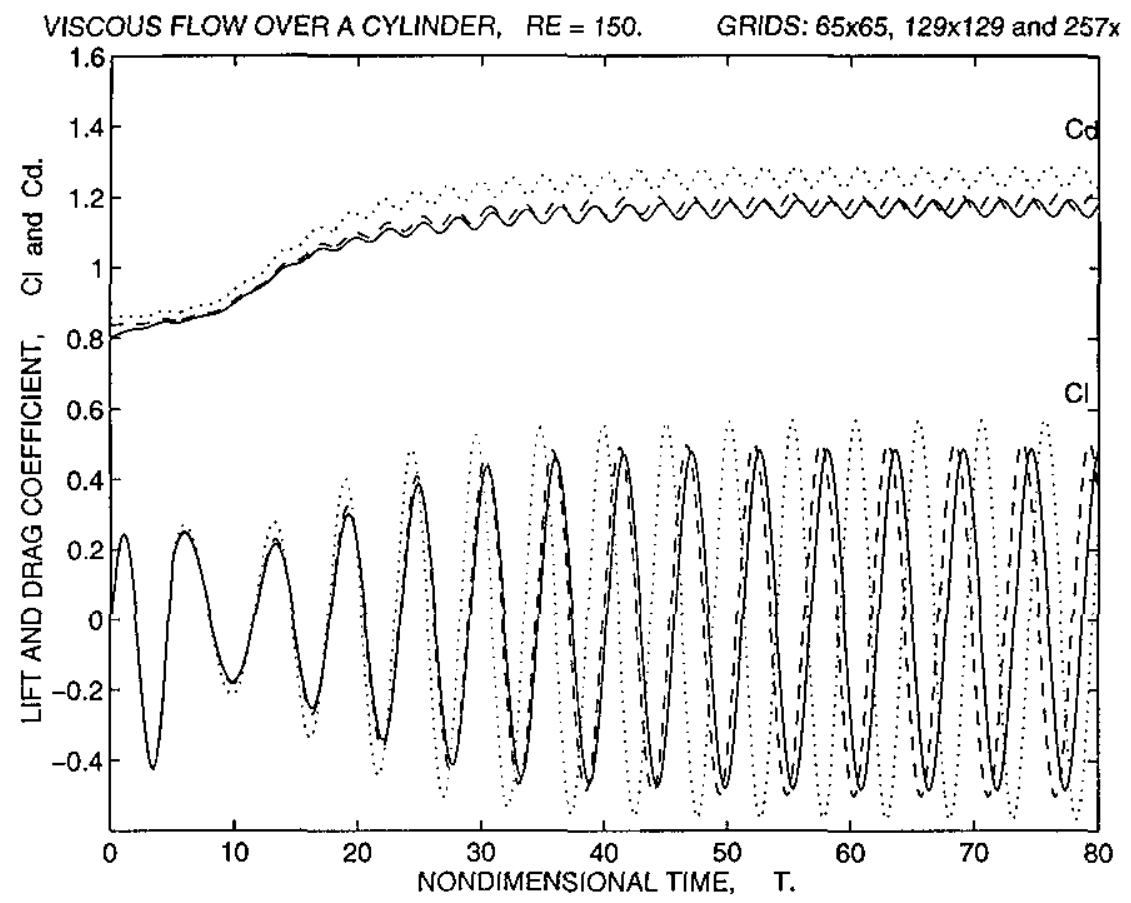

Figure 9: Flow over a Circular Cylinder: Grid Refinement Study.

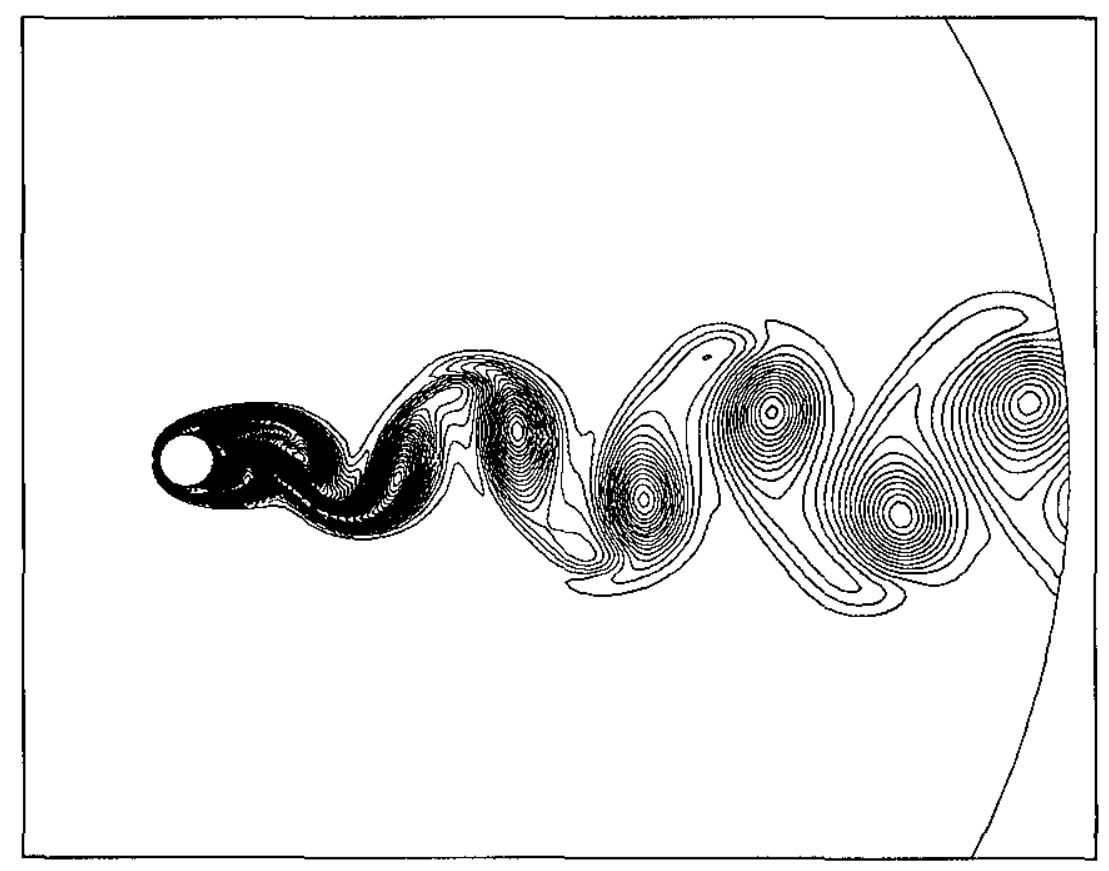

Figure 10: Vorticity Distribution in the Wake of a Circular Cylinder at $\mathrm{t}=62.875, R e=150$. 

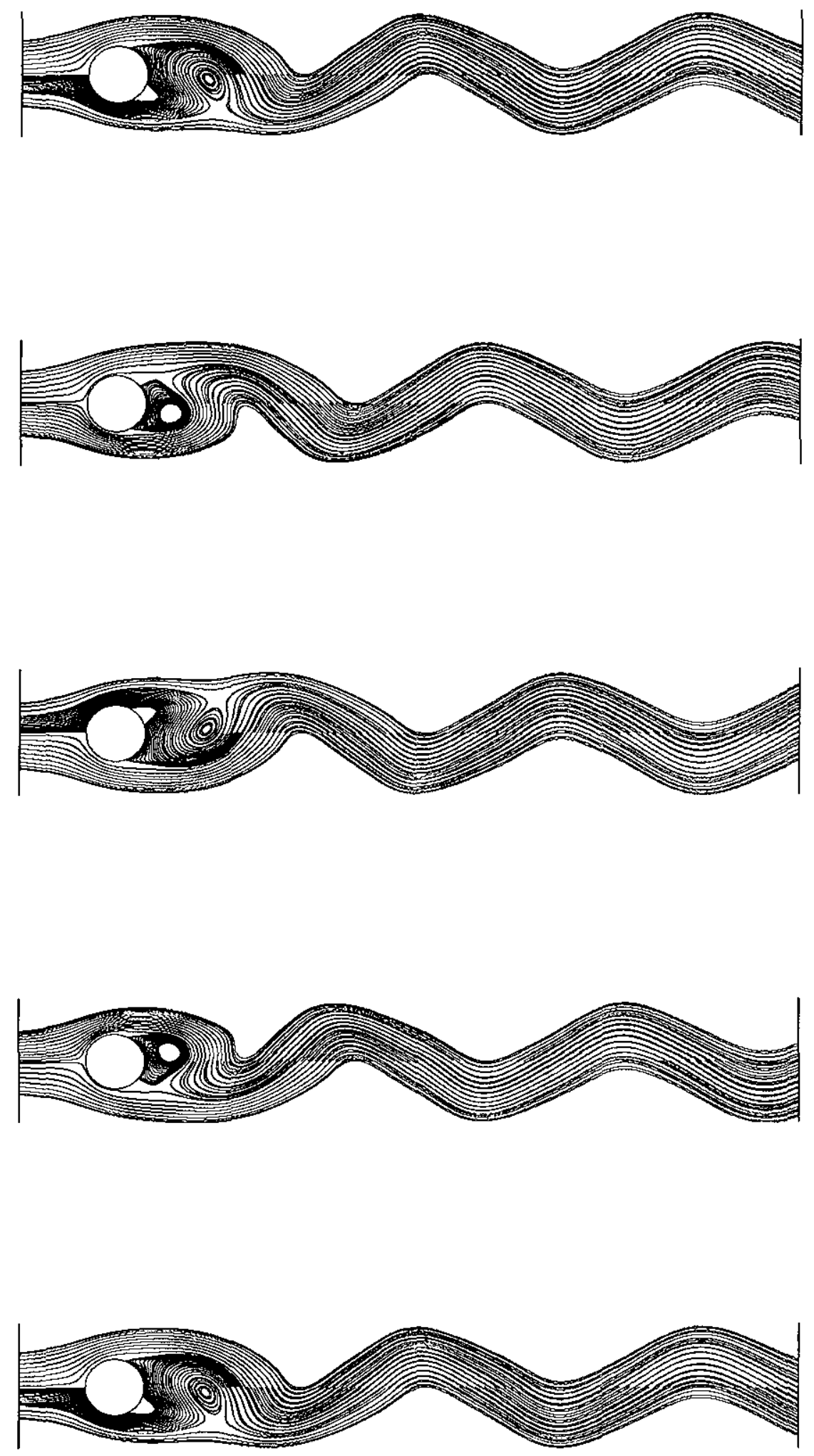

Figure 11: Parallel Vortex Shedding from a Circular Cylinder, $R e=150$. Nondimensional Time, $t=81.625$, $82.875,84.375,85.625$, and 87.125 


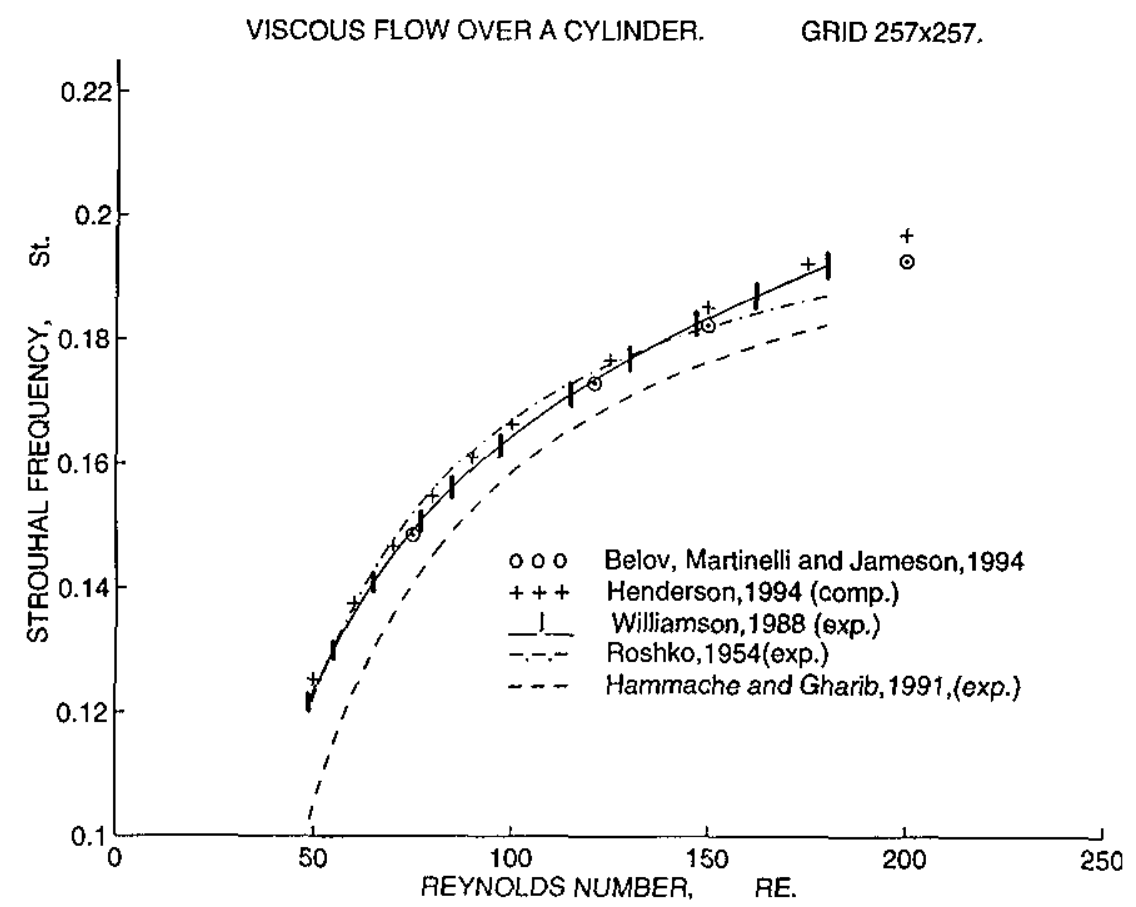

Figure 12: Variation of the Strouhal Frequency with Reynolds Number.

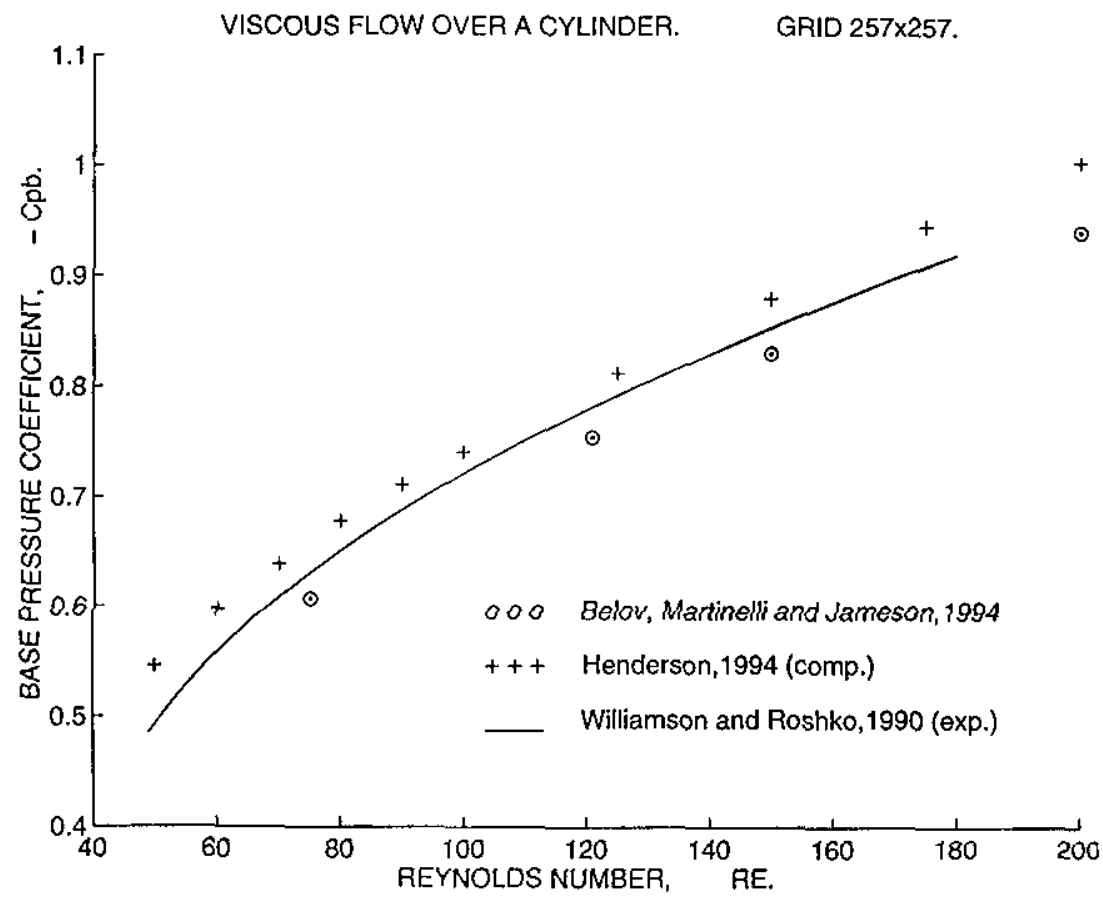

Figure 13: Variation of the Base Pressure Coefficient with Reynolds number. 


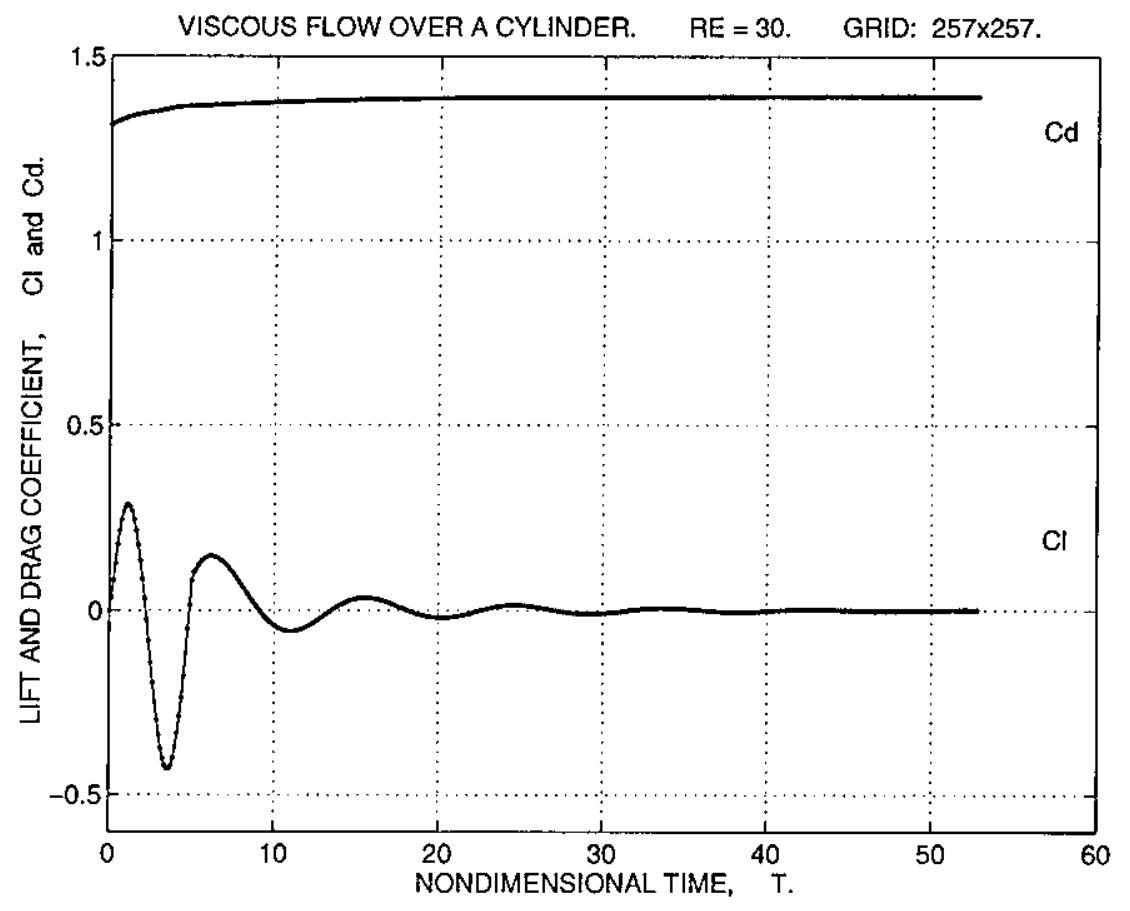

Figure 14: Decaying Response of the Wake of a Circular Cylinder, $R e=30$.

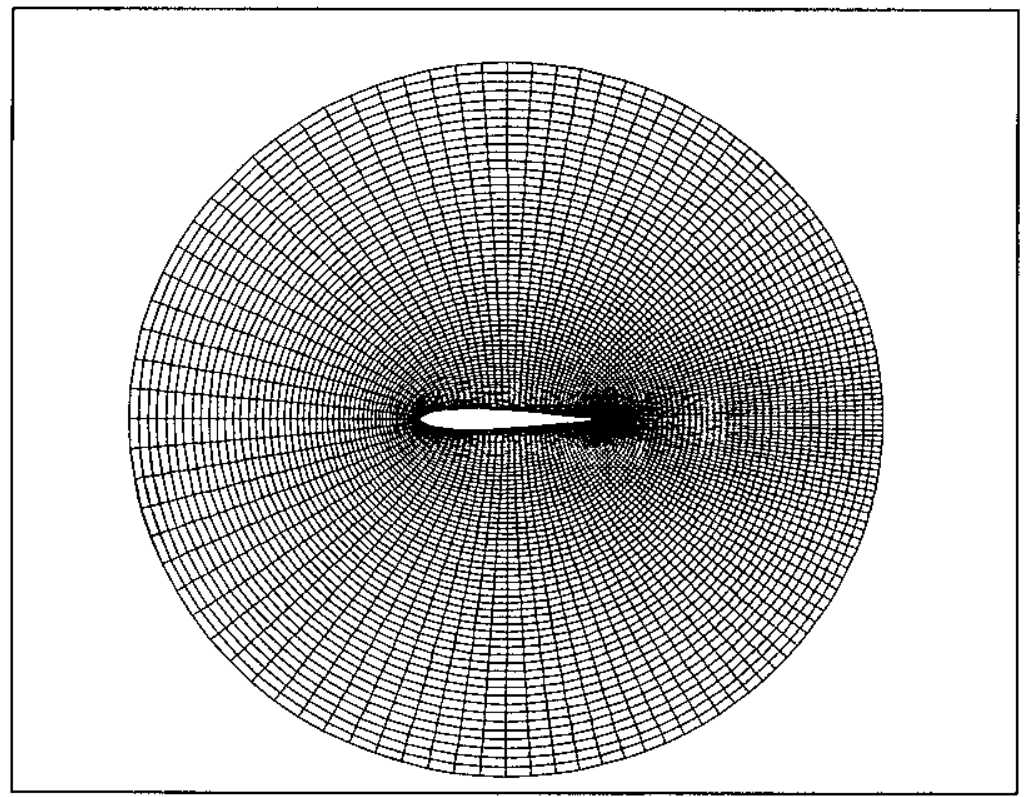

Figure 15: Detail of an O-Mesh. 


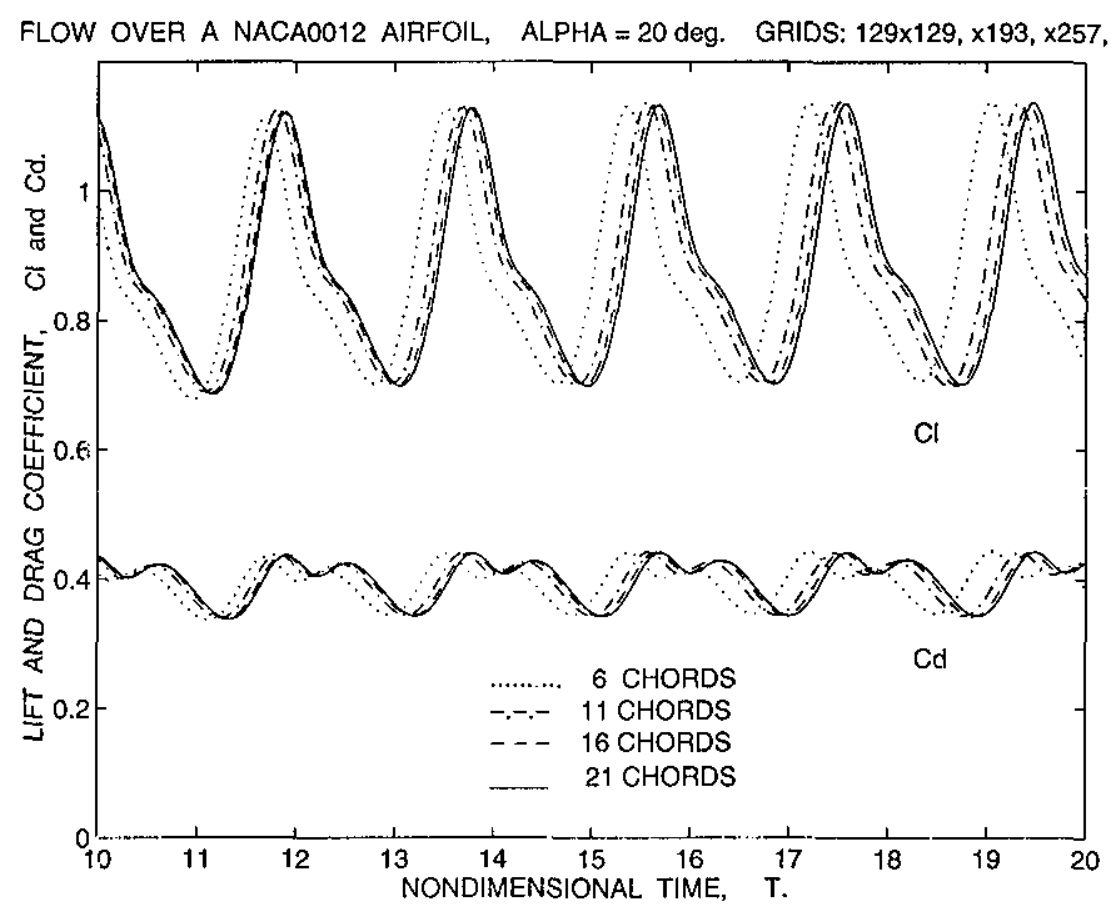

Figure 16: Evolution of the Drag and the Lift Coefficients of the NACA0012 Airfoil: Fffects of The Domain Truncation.

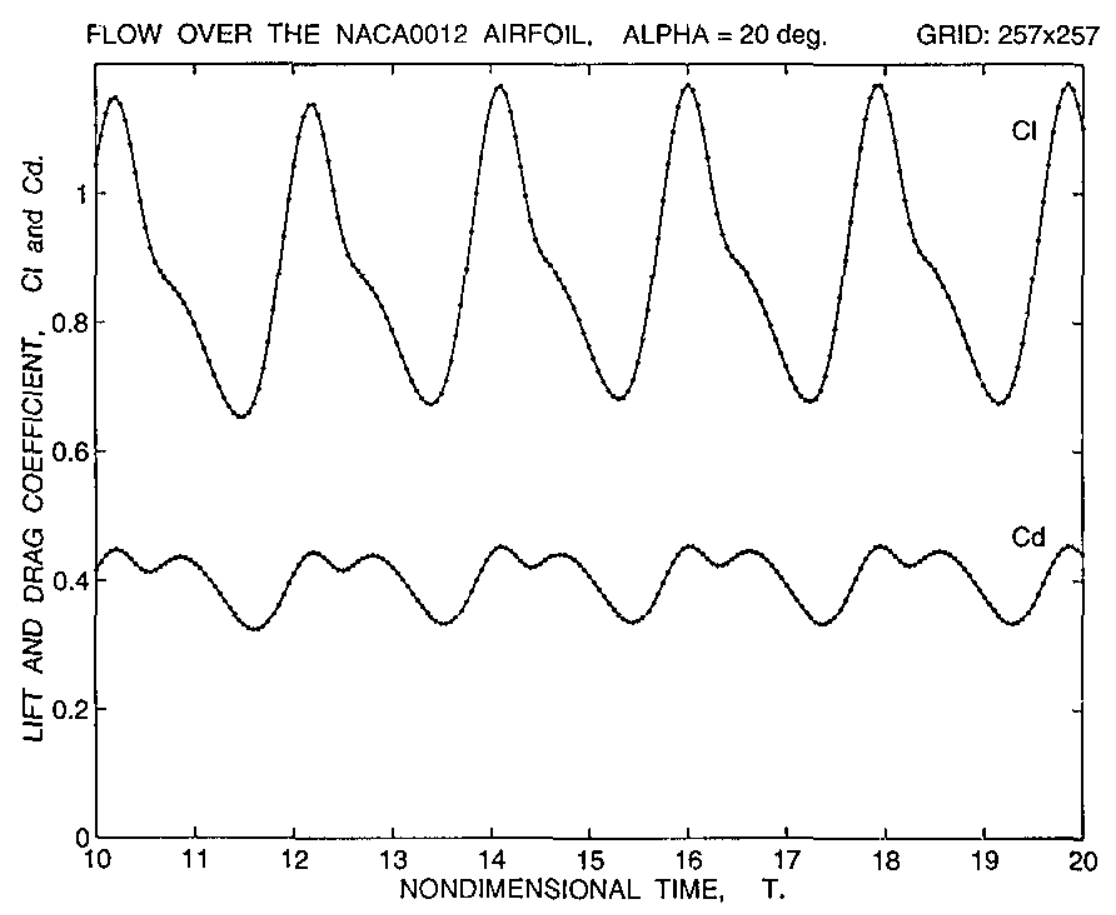

Figure 17: Evolution of the Aerodynamic Coefficients of the NACA0012 Airfoil, Re $=1000$. 


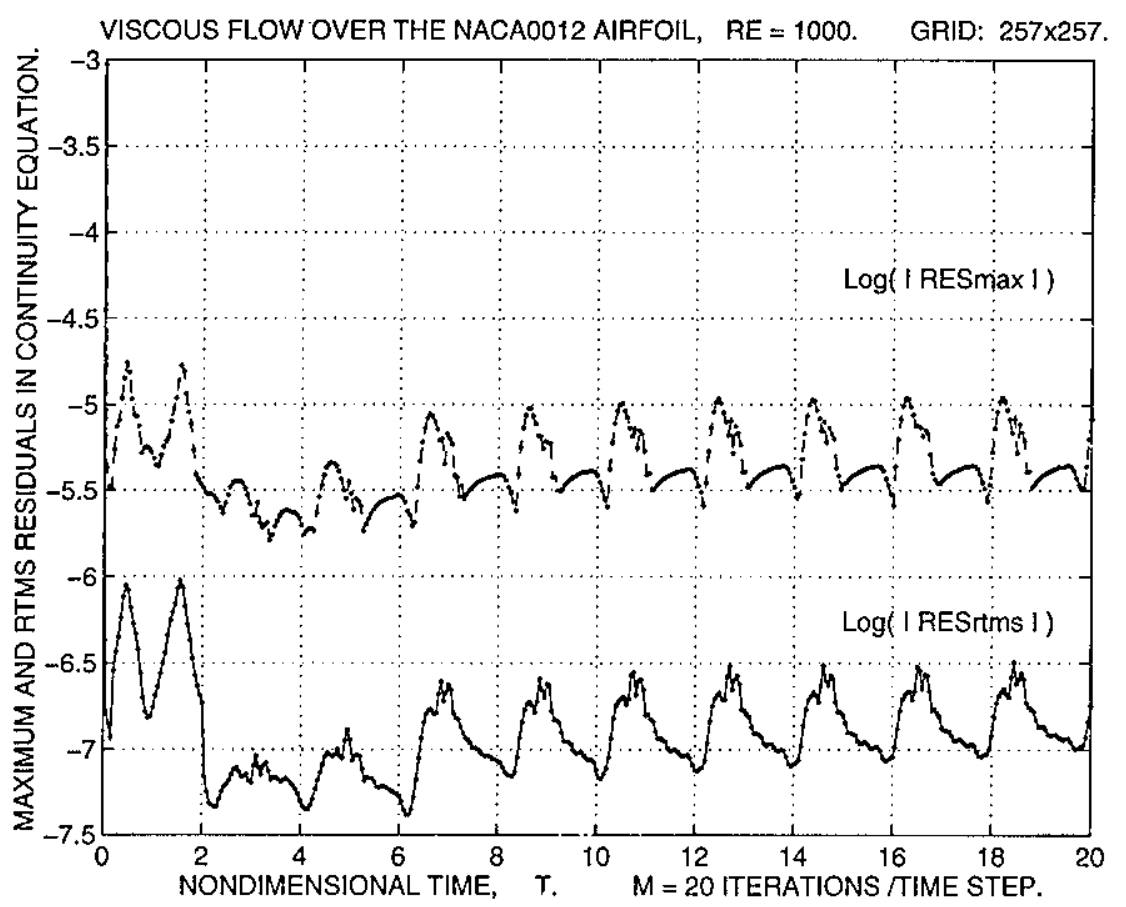

Figure 18: Contiruity Residuals Resulted on each Time Step after 20 Multigrid Cycles.

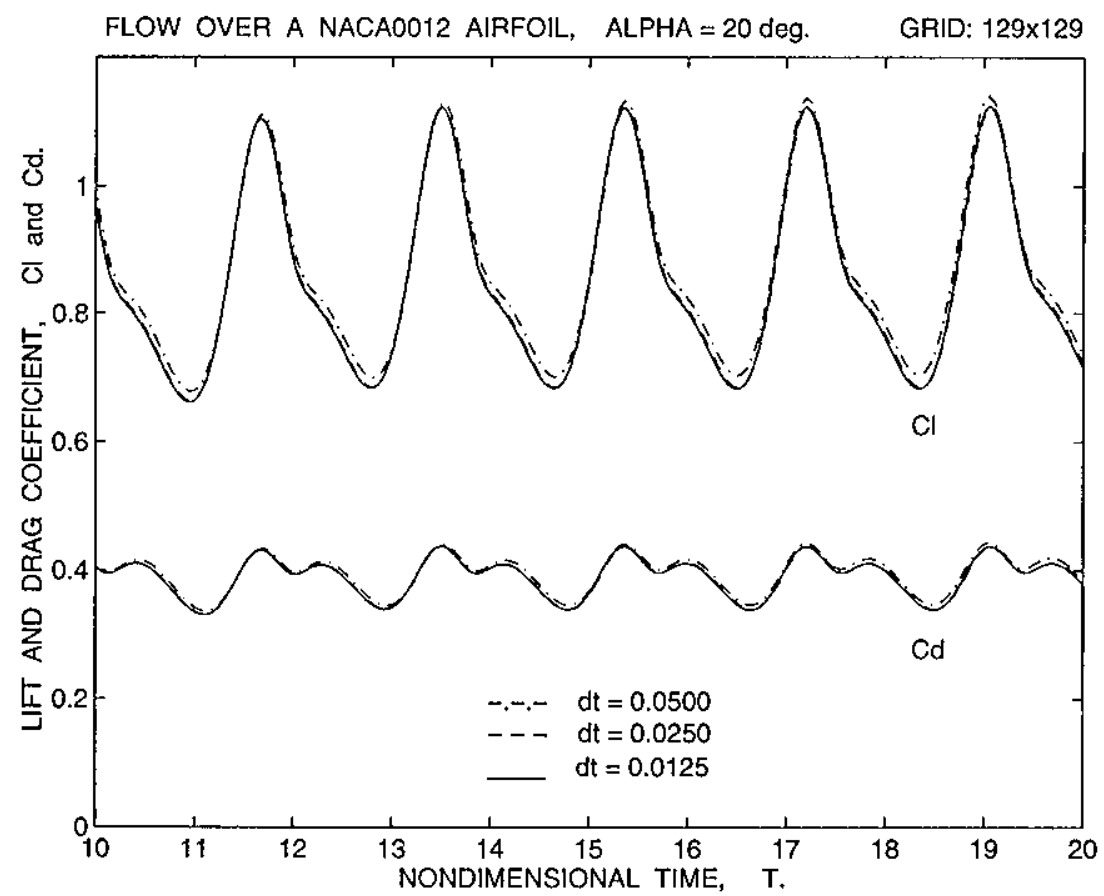

Figure 19: Evolution of the Aerodynamic Coefficients of the NACA0012 Airfoil: Time Resolution Study. 